\title{
Yale Autism Program's 8th Annual Summer Institute on Autism Spectrum Disorders July 25-27th, 2011
}

\author{
Yale Child Study Center, Yale University School of Medicine, New Haven, CT
}

(C) Springer Science+Business Media, LLC 2012

Objectives This Institute will provide $2 \frac{1}{2}$ days of specialized training on autism spectrum disorders, including the following topics:

- clinical features of ASDs

- diagnostic evaluation and differentiation

- early detection and intervention

- special education and consultation

- evidence-based social, communication, behavioral, and vocational interventions

- state of the art research paradigms in ASD
Participants This Institute will be beneficial for all professionals involved in the care of individuals with ASD in a clinical, medical, or educational setting, including Psychologists, Psychiatrists, Speech Pathologists, Occupational Therapists, Physical Therapists, Social Workers, Special Education professionals, Teachers, Paraprofessionals, Early Intervention Providers, Pediatricians, Physicians, Neurologists, Nurses, and Geneticists.

Contact Brian Reichow, PhD (brian.reichow@yale.edu) Website www.autism.fm 